\title{
Neurodevelopmental Disorders Caused by De Novo Variants in KCNB1 Genotypes and Phenotypes
}

\author{
Carolien G. F. de Kovel, PhD; Steffen Syrbe, MD, PhD; Eva H. Brilstra, MD, PhD; Nienke Verbeek, MD, PhD; \\ Bronwyn Kerr, PhD; Holly Dubbs, MD; Allan Bayat, MD, PhD; Sonal Desai, MGC, CGC; Sakkubai Naidu, MD; \\ Siddharth Srivastava, MD; Hande Cagaylan, MD; Uluc Yis, MD, PhD; Carol Saunders, PhD; Martin Rook, PhD; \\ Susanna Plugge, MSc; Hiltrud Muhle, MD; Zaid Afawi, MD, PhD; Karl-Martin Klein, MD, PhD; \\ Vijayakumar Jayaraman, MSc; Ramakrishnan Rajagopalan, PhD; Ethan Goldberg, MD, PhD; Eric Marsh, MD, PhD; \\ Sudha Kessler, MD, MSCE; Christina Bergqvist, MD; Laura K. Conlin, PhD; Bryan L. Krok, PhD; \\ Isabelle Thiffault, PhD; Manuela Pendziwiat, MSc; Ingo Helbig, MD; Tilman Polster, MD, PhD; \\ Ingo Borggraefe, MD, PhD; Johannes R. Lemke, MD, PhD; Marie-José van den Boogaardt, MD, PhD; \\ Rikke S. Møller, MSc, PhD; Bobby P. C. Koeleman, PhD
}

IMPORTANCE Knowing the range of symptoms seen in patients with a missense or loss-of-function variant in KCNB1 and how these symptoms correlate with the type of variant will help clinicians with diagnosis and prognosis when treating new patients.

OBJECTIVES To investigate the clinical spectrum associated with KCNB1 variants and the genotype-phenotype correlations.

DESIGN, SETTING, AND PARTICIPANTS This study summarized the clinical and genetic information of patients with a presumed pathogenic variant in KCNB1. Patients were identified in research projects or during clinical testing. Information on patients from previously published articles was collected and authors contacted if feasible. All patients were seen at a clinic at one of the participating institutes because of presumed genetic disorder. They were tested in a clinical setting or included in a research project.

MAIN OUTCOMES AND MEASURES The genetic variant and its inheritance and information on the patient's symptoms and characteristics in a predefined format. All variants were identified with massive parallel sequencing and confirmed with Sanger sequencing in the patient. Absence of the variant in the parents could be confirmed with Sanger sequencing in all families except one.

RESULTS Of 26 patients ( 10 female, 15 male, 1 unknown; mean age at inclusion, 9.8 years; age range, 2-32 years) with developmental delay, 20 (77\%) carried a missense variant in the ion channel domain of $K C N B 1$, with a concentration of variants in region $\mathrm{S} 5$ to $\mathrm{S} 6$. Three variants that led to premature stops were located in the C-terminal and 3 in the ion channel domain. Twenty-one of 25 patients (84\%) had seizures, with 9 patients (36\%) starting with epileptic spasms between 3 and 18 months of age. All patients had developmental delay, with 17 (65\%) experiencing severe developmental delay; 14 (82\%) with severe delay had behavioral problems. The developmental delay was milder in 4 of 6 patients with stop variants and in a patient with a variant in the S2 transmembrane element rather than the S4 to S6 region.

CONCLUSIONS AND RELEVANCE De novo KCNB1 missense variants in the ion channel domain and loss-of-function variants in this domain and the C-terminal likely cause neurodevelopmental disorders with or without seizures. Patients with presumed pathogenic variants in $K C N B 1$ have a variable phenotype. However, the type and position of the variants in the protein are (imperfectly) correlated with the severity of the disorder.
Author Affiliations: Author affiliations are listed at the end of this article.

Corresponding Author: Bobby P. C. Koeleman, PhD, Department of Genetics, University Medical Center Utrecht, PO Box 85500, 3508 GA Utrecht, the Netherlands (b.p.c.koeleman@umcutrecht.nl). 
$\mathrm{E}$ arly infantile epileptic encephalopathies (EEs) form a group of disorders that are characterized by epileptic seizures starting during the first year of life. Patients have developmental delay (DD) or even regression to which the epileptic discharges are presumed to contribute. The seizures are often unresponsive to treatment. Many of those epilepsies have a genetic disposition. ${ }^{1}$

In the past few years, several new genes have been identified that can cause EE when mutated. One of the more recently discovered genes is KCNB1 (potassium voltage-gated channel subfamily B member 1 or delayed rectifier potassium channel 1 [DRK1]) (OMIM 616056), which acts in a dominant manner. Variants are described by Torkamani et $\mathrm{al}^{2}{ }^{2}$ who identified a de novo KCNB1 variant when exome sequencing a family with a child with EE. They subsequently found 2 other independent patients with EE and KCNB1 variants. One of those patients had earlier been described in the Epi4K effort ${ }^{3}$ as having Lennox-Gastaut syndrome. Subsequently, more patients with variants in KCNB1 were described. ${ }^{4-10}$

The KCNB1 gene encodes the Kv2.1 pore-forming, voltagesensing a-subunit of a delayed rectifier potassium channel. It is expressed in various neuronal cells in the brain. ${ }^{11}$ In Ensembl (GRCh38.p3), only 1 transcript is known (ENST00000371741). It consists of only 2 exons and contains 4 known domains (Figure): the T1 domain, which is involved in multimerization; the ion_trans domain, which contains the transmembrane elements; and 2 intracellular Kv2 channel-specific domains (Pfam; http://smart.embl-heidelberg.de/). ${ }^{12,13}$ The protein has 6 transmembrane elements in the ion_trans domain; the first 4 form the voltage-sensor domain, with $S 4$ being the actual voltage sensor, whereas S5 and S6 form the pore domain of the protein. The extracellular loop between S5 and S6 contains the selectivity filter (TVGYG amino acids; UNIPROT.org). This structure is similar to other voltagegated potassium channels, such as KCNQ2 (OMIM 602235) and

\section{Key Points}

Question How does the phenotype of patients with a KCNB1 mutation correlate with the type of mutation?

Findings This study found that patients with a de novo mutation in $K C N B 1$ present a variable phenotype of neurodevelopmental disorder.

Meaning De novo mutations in $K C N B 1$ are associated with a variable neurodevelopmental disorder.

KCNQ3 (OMIM 602232). ${ }^{14}$ The protein forms homotetramers and heterotetramers with various other voltage-gated potassium channel proteins, such as KCNB2 (OMIM 607738), and proteins from the KCNG (Kv6), KCNE, and KCNS (Kv9) families. ${ }^{15-17}$

\section{Methods}

In a recent screen of 358 children with EEs, we identified 3 novel KCNB1 variants (approximately 1\%), all of which were found to be de novo. ${ }^{18}$ We subsequently collected data from 13 additional patients from research and diagnostic sources who were reported to carry a de novo novel KCNB1 variant not present in public databases. In this article, we describe the phenotypic and genetic characteristics of these patients. In addition, we collected more information about 10 patients who were described previously. For 3 of the 10 patients in previously published articles, the authors have provided updated information for the current article. We present the available information on 26 patients for a broad overview of the phenotypic and genetic variability and similarities. Statistical tests were performed using $x^{2} 2 \times 2$ contingency tables; the significance of the enrichment of de novo mutation was calculated using a

Figure. Distribution of Pathogenic Variants in KCNB1

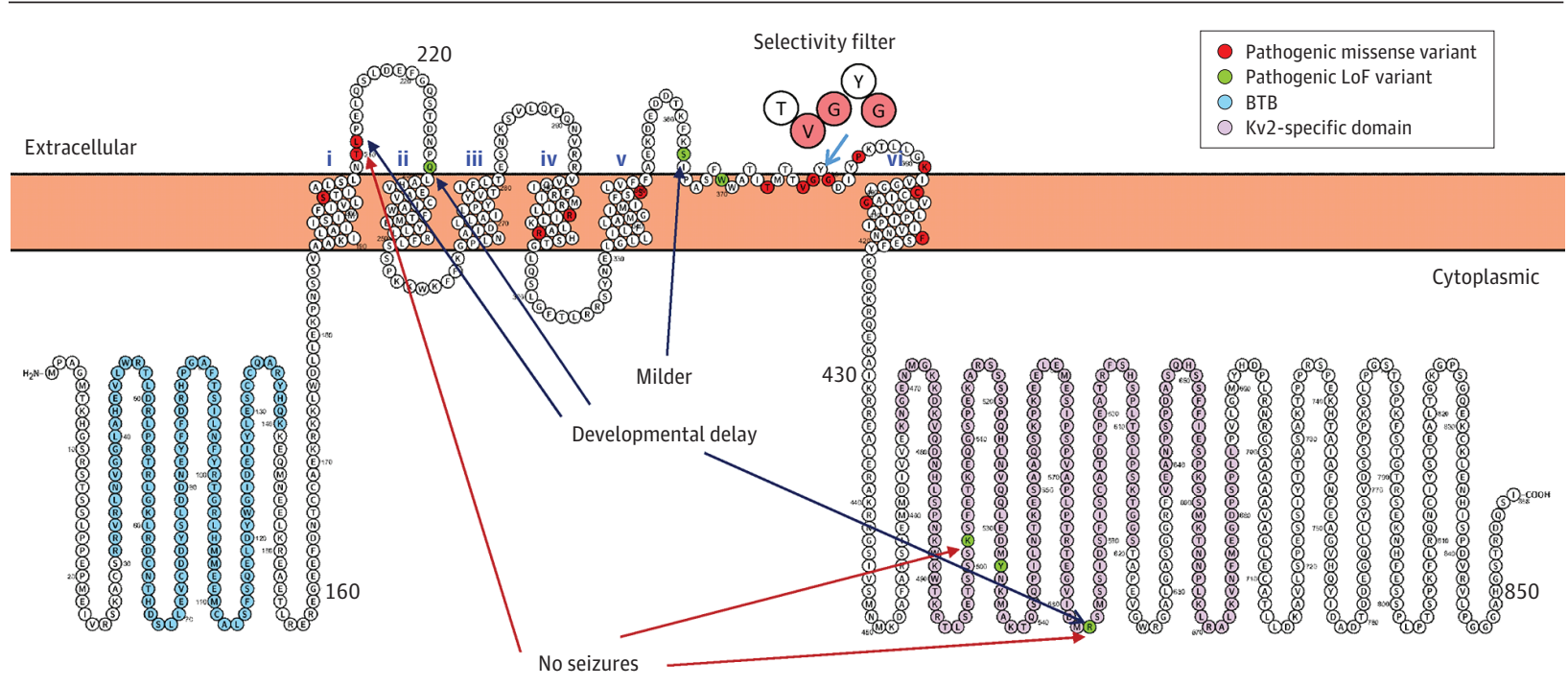

Schematic view of the Kv2.1 (KCNB1) protein in the membrane, showing pathogenic missense and loss-of-function (LoF) variants. BTB indicates broad-complex, tramtrack, and bric-brac domain. 
$\mathrm{X}^{2}$ goodness-of-fit test. A 2-sided $\chi^{2}$ test with $P<.05$ determined statistical significance. Collection of data procedures agreed with the local ethics guidelines at the different institutes.

\section{Results}

\section{Description of the Variants}

We identified 16 patients with KCNB1 variants who were previously not described and collected information about 10 previously described patients (10 female, 15 male, 1 unknown; mean age at inclusion, 9.8 years; age range, 2-32 years) ${ }^{2-9}$ In these 26 patients, we identified 23 different variants; 3 variants were seen twice each. Twenty patients (77\%) carried missense variants, and 6 (23\%) carried nonsense or frameshift variants leading to a premature termination codon (Table 1). All the missense variants are located within the Pfam ion_trans domain (ie, the 6 transmembrane regions and their extracellular and intracellular connecting loops) (Figure). Two variants in 4 patients are gating charge residues in voltage sensor $\mathrm{S} 4 .{ }^{19}$ Three variants are in the selectivity filter between S5 and S6. Three of the loss-of-function (LoF) variants are in the first Kv2 channel-specific domain in the intracellular C-terminus of the protein. The other $3 \mathrm{LoF}$ variants are in the S1 to S2 linker and in the S5 to S6 P-loop. The gene has 2 exons, and all variants are in the second (last) exon. All nonsense or frameshift variants are expected to result in a truncated protein rather than nonsense-mediated decay because they are located in the terminal part of the gene. No variants were detected in the cytoplasmic N-terminal part of the gene. All missense variants alter strongly conserved amino acids and are predicted to be highly deleterious by SIFT (Sorting Intolerant From Tolerant), Polyphen2, and Combined Annotation Dependent Depletion (CADD) scores. ${ }^{20-22}$ The lowest CADD score was 23.3. In all individuals except one (patient 18), for whom parental samples were not available for segregation analysis, the variant was confirmed to be de novo. The variant not confirmed to be de novo, $\mathrm{R} 312 \mathrm{H}$, was seen as de novo in another patient and was absent from the Exome Aggregation Consortium ExAC Browser (http://exac.broadinstitute.org/233; thus, we interpreted it to be pathogenic and included patient 18 in our series. An overview of characteristics of the patients and their variants is given in Table 1.

\section{Description of the Patients}

The observed phenotypes in patients are summarized in Table 2 and described in detail in this section. All 26 patients presented with primary DD in the first year of life, preceding onset of epilepsy. First signs of DD were unspecific, with muscular hypotonia or hypertonia being recurrently reported (11 of 23 individuals [48\%] with available neurologic examination findings). Twenty-one of 25 patients (84\%) had intractable epilepsy, with seizure onset in infancy or early childhood (median age at onset, 12 months; range, 3 months to 4 years). Epileptic spasms alone or as part of West syndrome were observed in 9 patients (36\%), with a median onset of seizures of 12 months (range, 3-18 months). The only patient who developed spasms before 6 months of age was patient 10 , who carried the prominent variant G381R within the selectivity filter of the P-loop. ${ }^{7}$ In most patients with epilepsy, the semiologic features developed over time into multiple seizure types, including tonic, focal-clonic, myoclonic, and atypical absences. Three patients did not have epileptic seizures (patients 1, 3, and 24), 1 reported only infantile spasms (patient 2 ), and no data were available for 1 (patient 8 ). The patients with LoF variants in the C-terminal region had no seizures or had infantile spasms only; in addition, 1 patient with a variant in the S1 to S2 linker reported no seizures. However, one patient (patient 3) with LoF variant in the C-terminus reported severe $\mathrm{DD}$. Of the patients with LoF variants, 2 were reported to have severe DD and 4 had moderate or moderate-severe DD (1 unspecified). In contrast, the only patient with a missense variant for whom moderate DD was reported was patient 23, who had a missense variant in the S1 to S2 linker. In contrast, all patients with severe epilepsy harbored variants within the voltage sensor (S4) and the pore-forming domains (S5, P-loop, and S6) of KCNB1. These observations suggest an association between location of mutation and phenotype and severity of disease.

In general, the epilepsy in most patients was highly refractory to diverse and multiple antiepileptic drugs. Anecdotally, partial efficacy has been reported for ketogenic diet, which resulted in a significant decrease in seizure burden in 2 patients (patients 14 and 18) but seemed ineffective in 2 others (patients 11 and 20). Improvement was also observed in 1 patient who received steroid pulses (patient 17), whereas intramuscular adrenocorticotropic hormone therapy for epileptic spasms was reported as not being of value in 3 patients (patients 2, 11, and 14). Vagus nerve stimulation and corpus callosotomy surgery were performed in 1 patient (patient 9) without substantial improvement of epilepsy. Brain biopsy in this patient revealed minimal cortical dysplasia consisting of excessive but not dysplastic neurons, a finding that argues against a role of $K C N B 1$ in disorders of neuronal migration.

Electroencephalographic (EEG) data were reported for 21 patients. The EEG displayed features of EE in 20 of 21 patients (95\%), including hypsarrhythmia and general slowing of background activity together with multifocal spikes and polyspikes, and 9 of 21 patients (43\%) with abnormal EEG results had a combination of focal and generalized epileptic discharges, with 1 being mainly focal. Five patients (24\%) had continuous spike and wave during slow-wave sleep (CSWS) or electrical status epilepticus during slow-wave sleep symptoms. Patients 14, 18, and 19 had CSWS on EEG, and patients 21 and 25 had a history of electrical status epilepticus during slow-wave sleep.

For 13 patients, more detailed information on motor function was available. Motor performance was poor, with limited ability to walk freely and significant signs of upper motor neuron disease (brisk reflexes, contractures, clonus, and positive plantar response) or ataxia in 10 patients (77\%), all with missense variants within vital channel domains (S4-S6). The remaining 3 patients had normal neurologic examination findings and carried variants in S2 or the C-terminal.

For 14 of 17 patients ( $82 \%$ ) with available data, behavioral problems were reported. Autism spectrum disorder was 


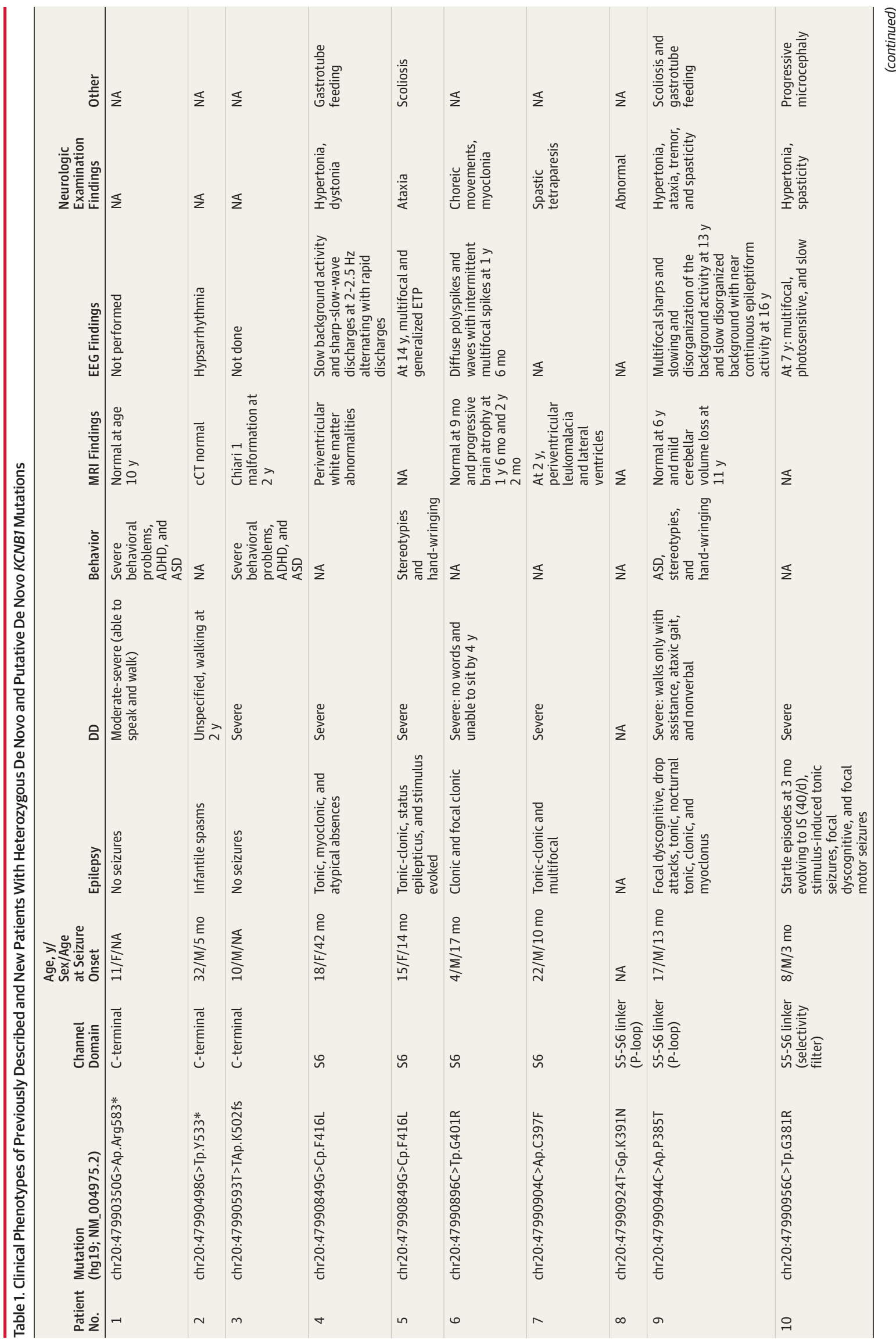




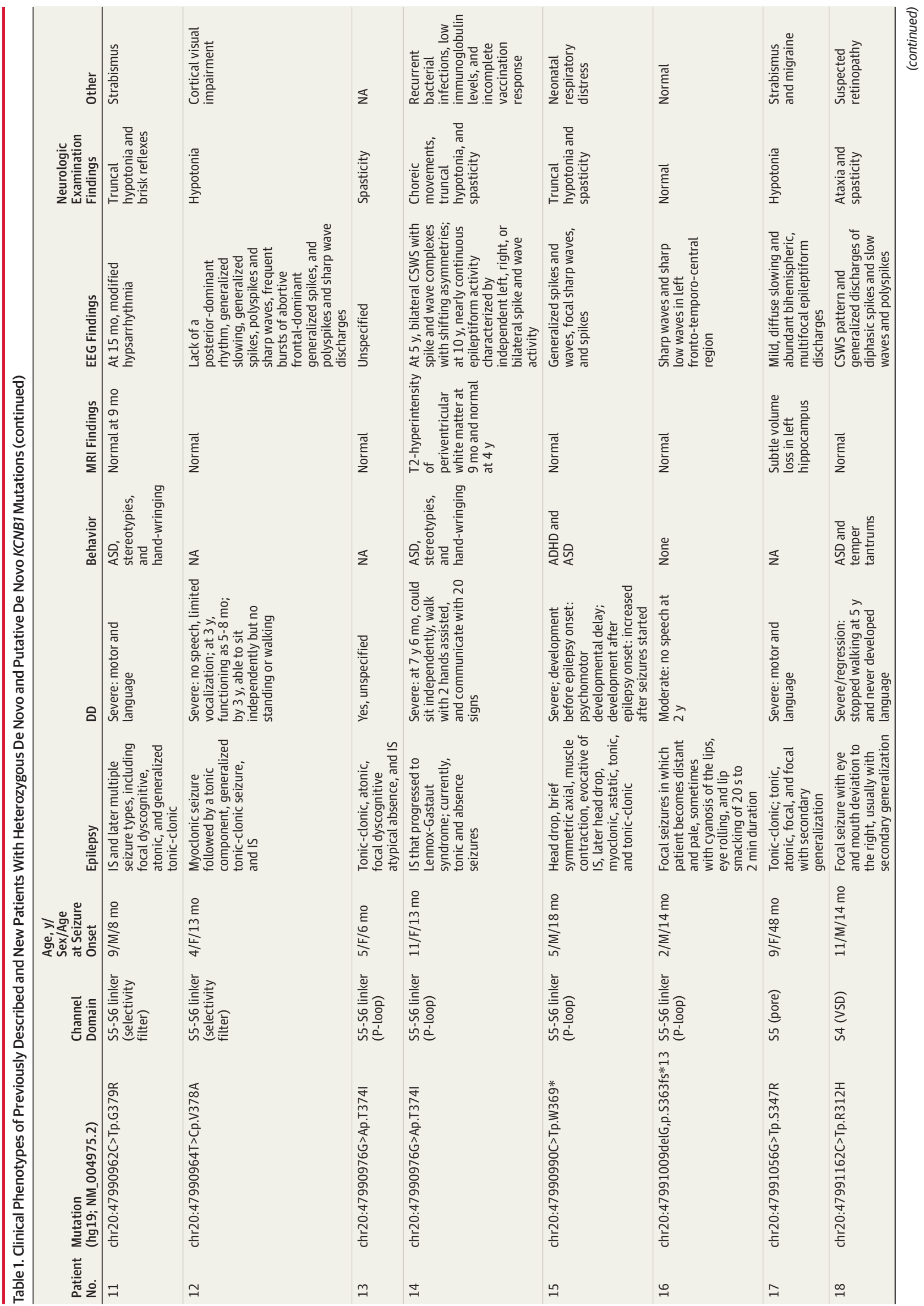




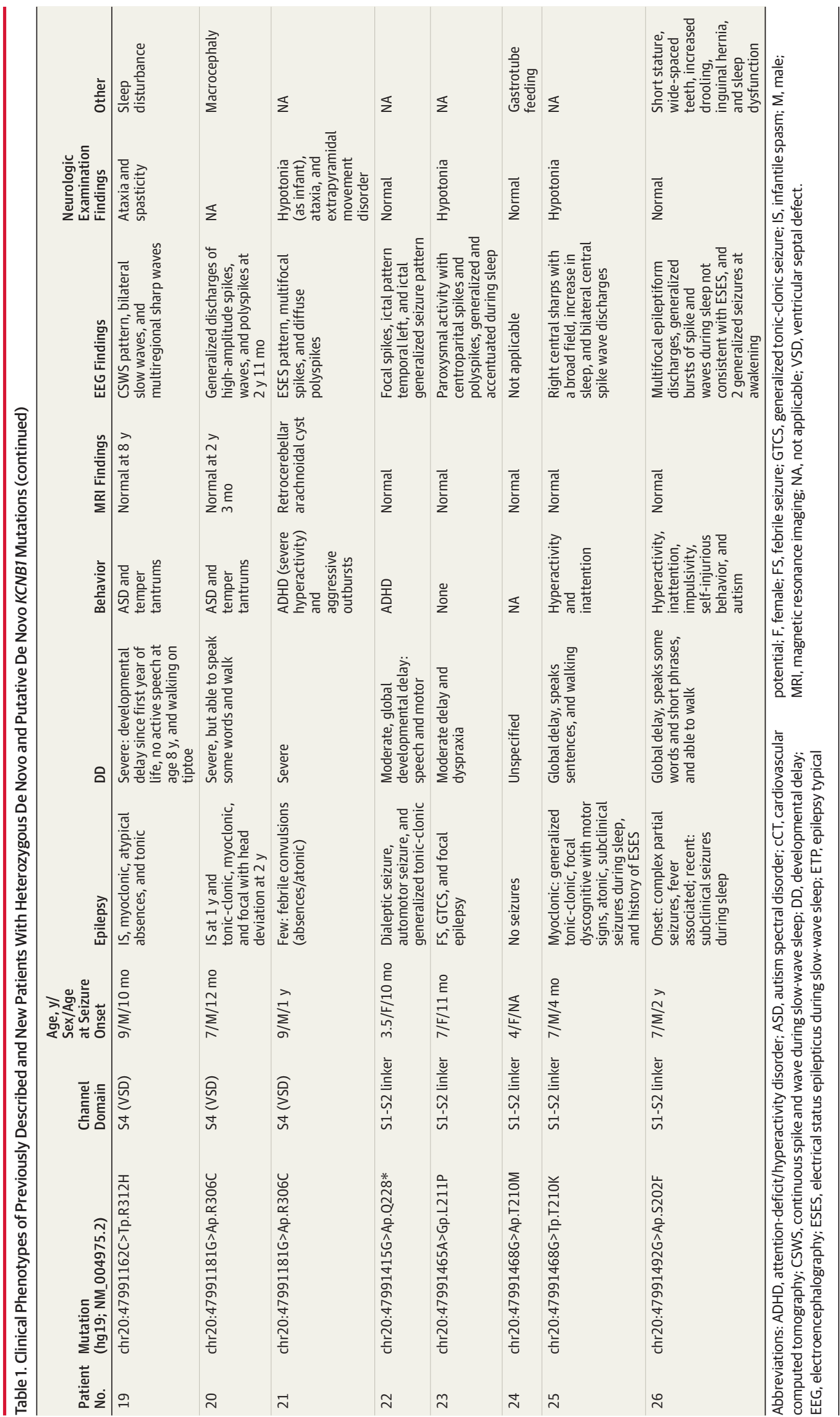


Table 2. Major Clinical Characteristics of the Patients Carrying a Presumed Pathogenic KCNB1 Mutation

\begin{tabular}{lc}
\hline Phenotype & $\begin{array}{l}\text { No. of Patients/Patients With } \\
\text { Data Available (\%) }\end{array}$ \\
\hline Developmental delay & $26 / 26(100)$ \\
\hline Intractable epilepsy & $21 / 25(84)$ \\
\hline Epileptic spasms & $9 / 25(36)$ \\
\hline EE-EEG & $20 / 21(95)$ \\
\hline Focal-generalized discharges & $9 / 21(43)$ \\
\hline CSWS or ESES & $5 / 21(24)$ \\
\hline Poor motor function & $10 / 13(77)$ \\
\hline Hypotonia & $11 / 23(48)$ \\
\hline Ataxia & $4 / 23(17)$ \\
\hline Abnormal behavior & $14 / 17(82)$ \\
\hline ADHD & $7 / 20(35)$ \\
\hline ASD & $10 / 20(50)$ \\
\hline Hand-wringing & $4 / 20(20)$ \\
\hline Temper tantrums & $3 / 20(15)$ \\
\hline Abnormal brain imaging & $4 / 23(17)$ \\
\hline
\end{tabular}

Abbreviations: ADHD, attention-deficit/hyperactivity disorder; ASD, autism spectrum disorder; CSWS, continuous spike and wave during slow-wave sleep; EE, epileptic encephalopathy; EEG, electroencephalography; ESES, electrical status epilepticus during slow-wave sleep.

reported in 10 individuals, with different variants located through $\mathrm{S} 4$ to $\mathrm{S} 6$ and the C-terminus. In 4 patients, caregivers noted specifically nonpurposeful hand-wringing stereotypies. In addition, attention-deficit/hyperactivity disorder or hyperactivity with inattention in 7 patients and temper tantrums in 3 patients were mentioned.

Information on brain imaging was available from 23 of 26 patients ( 22 with magnetic resonance imaging and 1 with computed tomography). Results were normal in 19 patients (83\%) or revealed unspecific features after infancy, such as ArnoldChiari malformation type 1 (patient 3), progressive supratentorial brain atrophy (patient 6, G401R, with infantile epilepsy), cerebellar volume loss (patient 8, P385T, infantile epilepsy proceeding to Lennox-Gastaut syndrome), and white matter abnormalities (patient 14, T374I, West syndrome progressing to Lennox-Gastaut syndrome), corresponding to seizure burden in these patients.

\section{Discussion}

All missense variants clustered within the ion_trans domain, in which few natural segregating variants are found. On the exome variants server with data from more than 6500 individuals (http://evs.gs.washington.edu/EVS/), no coding variants were found in the ion_trans domain. In the ExAC database with more than 60000 individuals, 9 coding variants were present in this domain.

On the basis of the work of Samocha et al, ${ }^{24}$ we explored the frequency of missense variants in the ExAC database in the different regions of the gene relative to the neutral expectation to identify gene regions intolerant to missense variation. The ratio of missense to synonymous variants is 0.26 in the ion_trans domain; 0.33 in the upstream region, including the T1 domain; and 2.14 in the downstream region, including the Kv2 channel-specific domains. This ratio is expected to be 2.3 for the whole gene after neutral expectation according to Samocha et $\mathrm{al}^{24}$; therefore, both the ion_trans domain and the upstream region of the gene are intolerant to missense variants $\left(P<1 \times 10^{-5}\right)$. Missense variants in those regions thus have a high probability of being pathogenic. ${ }^{24}$ We assume that all missense variants discussed in the present study are causal for the neurodevelopmental disorder in the patient.

We also consider the LoF variants described in the present study to be pathogenic. In the ExAC database, only 2 rare LoF variants were found: p.R854* (flagged as dubious) and p.K776Qfs*23. These variants are located in the C-terminal part of the gene, closer to the terminal part than were the LoF variants found in the patients and beyond the Kv2 channelspecific domains (Figure). Therefore, it can be expected that these variants, if they exist, do not affect normal function of Kv2.1. In contrast, the $3 \mathrm{C}$-terminal mutations observed in the patients were in the Kv2.1-specific domain located more upstream and therefore may be expected to have a functional effect, for example, lead to differences in protein folding or affect formation of tetramers. All LoF variants in the patients were in the last exon of the gene; thus, we expect that they will lead to truncation rather than nonsense-mediated decay. ${ }^{25}$ Because the protein forms (hetero)tetramers, we expect that the effect of the missense and truncating variants is dominant negative unless the mutated protein is not incorporated in the membrane.

Because we consider all variants described in this article as causal for the phenotype, the phenotypic range described is likely to be representative of variants in this gene. There may be a bias, however, toward patients with epilepsy because our initial screen was conducted in a panel of patients with EE and many colleagues in our network have a special interest in this phenotype. Torkamani et $\mathrm{al}^{2}$ may also have searched only for patients with seizures when following up their initial finding. The patients without seizures in this article were from exomewide diagnostic screening of patients with (neuro)developmental problems and from the Deciphering Developmental Disorders program in the United Kingdom, which also was a general screening of children with developmental disorders. As described in the Results section and as seen in Table 1, the range of symptoms in patients with variants in KCNB1 was diverse in many aspects.

\section{Genotype-Phenotype Associations}

Most missense variants were in the S5 to S6 linker, which forms the pore region, particularly in or near the selectivity filter. Three patients had missense variants in one of the positively charged R-residues in voltage sensor S4. As described in the Results section, the distribution of variants in patients over the domains is different from that in the general population. The variants that we found in the less constrained C-terminus were LoF variants.

Missense variants within the voltage sensor domain or the pore region of the channel were found in patients with severe epilepsy and global DD, including intellectual disability and often spasticity. Therefore, missense variants in the S4 to S6 re- 
gion seem to be correlated with a more severe prognosis. An LoF variant close to $\mathrm{S} 2$ and one in the $\mathrm{C}$-terminus were found in patients with milder DD, who acquired walking and were able to communicate. Two of the $3 \mathrm{LoF}$ variants in the C-terminus were in patients without seizures (at 11 and 32 years of age), whereas a patient with a missense variant in the S1 to S2 linker, close to S1, had no seizures at 4 years of age. The degree of intellectual disability in this patient was unspecified. ${ }^{9}$ The LoF variant 15 (p.W369*) in the pore region was seen in a patient with severe $\mathrm{DD}$ and epilepsy at the age of 5 years, whereas the LoF variant near that in LoF variant 16 (p.S363fs*) was found in a patient at 2 years of age with moderate $\mathrm{DD}$ and focal seizures but no additional reported problems.

No pathogenic variants have been reported thus far in the highly constrained N-terminal region. A handful of rare missense variants were described for this region in the ExAC control population. It remains to be determined whether missense or LoF variants in this region also lead to neurodevelopmental disorders. The strong constraints on variation in this region suggest that many of the possible variants are deleterious or even fatal. Finally, patients carrying missense mutations more often had abnormal neurologic examination results (17 of 19 available [89\%]) compared with 1 of $3 \mathrm{LoF}$ carriers (33\%) $\left(P=.02, \chi^{2}\right.$ test for independence). Similarly, normal magnetic resonance imaging results were reported for all $6 \mathrm{pa}-$ tients carrying an LoF mutation. Overall, patients with KCNB1 mutations have a variable phenotype, a finding highlighted by the observation that even patients carrying the same mutation have differences in phenotype.

Functional studies ${ }^{2,8,9}$ have been performed for several of the described variants. In general, the electrophysiologic characteristics of the different variants have a variety of functional aberrations, such as loss of voltage sensing (p.R306C), reduced current (p.G410R), and suppression of repetitive firing (p.R306C, p.G410R). Of interest, all 3 missense mutations in the ion-selectivity filter domain have been studied and render Kv2.1, a nonselective cation channel that is not voltage activated. However, the general loss of channel function and dominant-negative effects seen in most studied variants are in support of the presumed pathogenicity and link to the EE phenotype. Finally, a Kv2.1 $1^{-/-}$mouse model was not prone to spontaneous seizures but exhibited hypocampal hyperexcitability and epileptiform activity on stimulation. ${ }^{26}$ Together, these observations further support our conclusion that the KCNB1 variants described here are likely to be pathogenic.

\section{Comparison to KCNQ2}

KCNB1 forms a gene family with KCNB2, with which it can form heterotetramers. To date, KCNB2 has not been implicated in monogenic disorders. KCNB1 has a similar structure as other potassium channels involved in severe epilepsy, such as $K C N Q 2$. Pathogenic mutations leading to EE in the other potassium channels are found in the transmembrane regions. This finding is similar to the mutations in KCNB1 described in this study. Furthermore, 2 of the pathogenic variants found in KCNQ2 27 were homologous to presumed pathogenic variants described in this article: the charged residue in the voltage sensor (p.R306C; variant 20) and missense variants 13 and 14 (p.T374I) in the pore region. This finding underlines the importance of these residues for the functioning of the proteins.

\section{Limitations}

Our study also has some limitations. Our patients may have been selected for certain phenotypes, in particular for epilepsy. Our focus on EE may have excluded cases with much milder phenotypes. Furthermore, the number of observations remain small, and description of additional cases will further elucidate the full clinical spectrum associated with mutations in KCNB1.

\section{Conclusions}

Missense and LoF variants in the ion_trans domain of KCNB1 and LoF variants in the C-terminal region can cause neurodevelopmental disorder with or without epileptic seizures. Missense variants in the C-terminus are less likely to cause developmental disorders, whereas the consequences of variants in the $\mathrm{N}$-terminus is not clear. The more severely affected patients consistently harbored missense variants within vital channel domains of KCNB1 and experienced late-onset epileptic spasms that evolved to treatment-refractive epilepsy and severe global DD. The effect of LoF variants was less clear, although a tendency existed toward less severe phenotypes. However, more outcome data on patients with LoF variants are needed to support clinical predictions and counseling of such patients. There was a marked tendency to develop spastic paraparesis or tetraparesis and ataxia as well as autistic spectrum disorder. Finally, patients carrying missense mutations were more likely to have neurologic and magnetic resonance imaging abnormalities.

\section{ARTICLE INFORMATION}

Accepted for Publication: May 18, 2017.

Published Online: August 14, 2017. doi:10.1001/jamaneurol.2017.1714

Author Affiliations: Department of Genetics, University Medical Center Utrecht, Utrecht, the Netherlands (de Kovel, Brilstra, Verbeek, van den Boogaardt, Koeleman); Department of Language and Genetics, Max Planck Institute for Psycholinguistics, Nijmegen, the Netherlands (de Kovel); Division of Child Neurology and Inherited Metabolic Diseases, Department of General Pediatrics, Center for Pediatrics and
Adolescent Medicine, University Hospital Heidelberg, Heidelberg, Germany (Syrbe); Institute of Evolution, Systems and Genomics, Faculty of Medical and Human Sciences, University of Manchester, Manchester, England (Kerr); Manchester Centre For Genomic Medicine, Central Manchester University Hospitals National Health Service Foundation Trust, Manchester, England (Kerr); Manchester Academic Health Science Centre, Manchester, England (Kerr); Division of Neurology, The Children's Hospital of Philadelphia, Philadelphia, Pennsylvania (Dubbs, Goldberg, Marsh, Kessler, Bergqvist, Helbig); Department of Pediatrics, University Hospital of Hvidovre,
Copenhagen, Denmark (Bayat); Department of Neurogenetics, Kennedy Krieger Institute, Baltimore, Maryland (Desai); Department of Neurology and Pediatrics, Johns Hopkins School of Medicine, Baltimore, Maryland (Naidu); Hugo Moser Research Institute, Kennedy Krieger Institute, Baltimore, Maryland (Naidu); Department of Neurology, Boston Children's Hospital, Boston, Massachusetts (Srivastava); Department of Molecular Biology and Genetics, Bogaziçi University, Istanbul, Turkey (Cagaylan); Division of Child Neurology, Department of Pediatrics, Schoo of Medicine, Dokuz Eylül University, İzmir, Turkey

(Yis); Center for Pediatric Genomic Medicine, 
Department of Pathology and Laboratory Medicine Children's Mercy Hospital, Kansas City, Missouri (Saunders, Thiffault); Department of Pediatrics, Children's Mercy Hospital, Kansas City, Missouri (Saunders, Thiffault); Pediatric Pathology and Laboratory Medicine, University of Missouri-Kansas City School of Medicine, Kansas City (Saunders); Department of Medical Physiology, University Medical Center Utrecht, Utrecht, the Netherlands. (Rook); Department of Biomedical Sciences, University Medical Center Utrecht, Utrecht, the Netherlands (Plugge); Department of Neuropediatrics, University Medical Center Schleswig-Holstein, Christian Albrechts University, Kiel, Germany (Muhle, Pendziwiat); Department of Physiology and Pharmacology, Tel Aviv University Medical School, Ramat Aviv, Israel (Afawi); Department of Neurology, Epilepsy Center Frankfurt Rhine-Main, Center of Neurology and Neurosurgery, University Hospital, GoetheUniversity Frankfurt, Frankfurt, Germany (Klein); Division of Genomic Diagnostics, Children's Hospital of Philadelphia, Philadelphia, Pennsylvania (Jayaraman, Rajagopalan, Conlin, Krok); University Medical Center Schleswig-Holstein, Christian Albrechts University, Kiel, Germany (Helbig); Epilepsiezentrum Bethel, Krankenhaus Mara, Kinderepileptologie, Bielefeld, Germany (Polster); Department of Pediatric Neurology, Developmental Medicine and Social Pediatrics Dr. von Hauner's Children's Hospital, University of Munich, Munich, Germany (Borggraefe); Institute of Human Genetics, University of Leipzig Hospitals and Clinics Leipzig, Germany (Lemke); Danish Epilepsy Centre, Dianalund, Denmark (Møller); Institute for Regiona Health Services, University of Southern Denmark, Odense, Denmark (Møller)

Author Contributions: Dr de Kovel had full access to all the data in the study and takes responsibility for the integrity of the data and the accuracy of the data analysis.

Study concept and design: de Kovel, Syrbe, Yis, Koeleman.

Acquisition, analysis, or interpretation of data: de Kovel, Syrbe, Brilstra, Verbeek, Kerr, Dubbs, Bayat, Desai, Naidu, Srivastava, Cagaylan, Saunders, Rook, Plugge, Muhle, Afawi, Klein, Rajagopalan, Jayaraman, Goldberg, Marsh, Kessler, Bergqvist, Conlin, Krok, Thiffault, Pendziwiat, Helbig, Polster, Borggraefe, Lemke, van den Boogaardt, Møller, Koeleman.

Drafting of the manuscript: de Kovel, Kerr, Plugge, Afawi, Jayaraman, Helbig, Koeleman. Critical revision of the manuscript for important intellectual content: de Kovel, Syrbe, Brilstra, Verbeek, Dubbs, Bayat, Desai, Naidu, Srivastava, Cagaylan, Yis, Saunders, Rook, Muhle, Klein, Rajagopalan, Goldberg, Marsh, Kessler, Bergqvist, Conlin, Krok, Thiffault, Pendziwiat, Helbig, Polster, Borggraefe, Lemke, van den Boogaardt, Møller, Koeleman.

Statistical analysis: de Kovel, Syrbe, Afawi. Obtained funding: Helbig, Koeleman.

Administrative, technical, or material support: de Kovel, Syrbe, Brilstra, Verbeek, Dubbs, Bayat, Desai, Naidu, Cagaylan, Yis, Rajagopalan, Goldberg Kessler, Bergqvist, Conlin, Thiffault, Helbig, Lemke, van den Boogaardt, Koeleman.

Study supervision: Afawi, Kessler, Helbig, Koeleman. Conflict of Interest Disclosures: None reported. Funding/Support: This study was funded by grant G.A.136.11.N from the Dutch Epilepsy Foundation
(Dr Koeleman) and Euroepinomics-RES (Dr Helbig). Dr Helbig was supported by grants HE 5415/5-1 and HE 5415/6-1 from the German Research Foundation and grant U01-HG006546 from the National Human Genome Research Institute of the National Institutes of Health. The Wellcome Trust supported the data generation of the Decipher project.

Role of the Funder/Sponsor: The sponsors had no role in the design and conduct of the study; collection, management, analysis, and interpretation of the data; preparation, review, or approval of the manuscript; and decision to submit the manuscript for publication.

Additional Contributions: We acknowledge the contributions of Nancy Spinner and Ian Krantz, who were not compensated for their help. This study makes use of data generated by the Database of Genomic Variation and Phenotype in Humans Using Ensembl Resources (DECIPHER) community. A full list of centers that contributed to the generation of the data is available at http://decipher.sanger.ac.uk and via email from decipher@sanger.ac.uk.We thank the patients and their parents for contributing to this research and all clinicians who provided samples from their patients. We thank the Exome Aggregation Consortium and the groups that provided exome variant data for comparison. A full list of contributing groups can be found at http://exac.broadinstitute.org/about.

\section{REFERENCES}

1. Deprez $L$, Jansen $A$, De Jonghe P. Genetics of epilepsy syndromes starting in the first year of life. Neurology. 2009;72(3):273-281.

2. Torkamani A, Bersell K, Jorge BS, et al. De novo KCNB1 mutations in epileptic encephalopathy. Ann Neurol. 2014;76(4):529-540.

3. Allen AS, Berkovic SF, Cossette P, et al; Epi4K Consortium; Epilepsy Phenome/Genome Project. De novo mutations in epileptic encephalopathies. Nature. 2013;501(7466):217-221.

4. Soden SE, Saunders CJ, Willig LK, et al. Effectiveness of exome and genome sequencing guided by acuity of illness for diagnosis of neurodevelopmental disorders. Sci Transl Med. 2014;6(265):265ra168.

5. Srivastava $\mathrm{S}$, Cohen JS, Vernon $\mathrm{H}$, et al. Clinical whole exome sequencing in child neurology practice. Ann Neurol. 2014;76(4):473-483.

6. Deciphering Developmental Disorders Study. Large-scale discovery of novel genetic causes of developmental disorders. Nature. 2015;519(7542) 223-228.

7. Allen NM, Conroy J, Shahwan A, et al. Unexplained early onset epileptic encephalopathy: exome screening and phenotype expansion. Epilepsia. 2016;57(1):e12-e17.

8. Thiffault I, Speca DJ, Austin DC, et al. A novel epileptic encephalopathy mutation in KCNB1 disrupts Kv2.1 ion selectivity, expression, and localization. J Gen Physiol. 2015;146(5):399-410.

9. Saitsu H, Akita T, Tohyama J, et al. De novo KCNB1 mutations in infantile epilepsy inhibit repetitive neuronal firing. Sci Rep. 2015;5:15199.

10. Latypova X, Matsumoto N, Vinceslas-Muller C, Bézieau S, Isidor B, Miyake N. Novel KCNB1 mutation associated with non-syndromic intellectual disability. J Hum Genet. 2017;62(5): 569-573.
11. Bishop HI, Guan D, Bocksteins E, et al. Distinct cell- and layer-specific expression patterns and independent regulation of Kv2 channel subtypes in cortical pyramidal neurons. J Neurosci. 2015;35 (44):14922-14942.

12. Letunic I, Doerks T, Bork P. SMART: recent updates, new developments and status in 2015. Nucleic Acids Res. 2015;43(database issue): D257-D260.

13. Finn RD, Bateman A, Clements J, et al. Pfam: the protein families database. Nucleic Acids Res. 2014:42(database issue):D222-D230.

14. Kuang $Q$, Purhonen $P$, Hebert $H$. Structure of potassium channels. Cell Mol Life Sci. 2015;72(19) 3677-3693.

15. Ruppersberg JP, Schröter KH, Sakmann B, Stocker M, Sewing S, Pongs O. Heteromultimeric channels formed by rat brain potassium-channel proteins. Nature. 1990;345(6275):535-537.

16. Ottschytsch N, Raes A, Van Hoorick D, Snyders DJ. Obligatory heterotetramerization of three previously uncharacterized Kv channel a-subunits identified in the human genome. Proc Natl Acad Sci U S A. 2002;99(12):7986-7991.

17. Bocksteins $E$, Mayeur E, Van Tilborg A, Regnier G, Timmermans JP, Snyders DJ. The subfamily-specific interaction between Kv2.1 and Kv6.4 subunits is determined by interactions between the N- and C-termini. PLoS One. 2014;9 (6):e98960.

18. de Kovel CG, Brilstra EH, van Kempen MJ, et al; EuroEPINOMICS RES Consortium. Targeted sequencing of 351 candidate genes for epileptic encephalopathy in a large cohort of patients. $\mathrm{Mol}$ Genet Genomic Med. 2016;4(5):568-580.

19. Islas LD, Sigworth FJ. Voltage sensitivity and gating charge in Shaker and Shab family potassium channels. J Gen Physiol. 1999;114(5):723-742.

20. Ng PC, Henikoff S. Predicting the effects of amino acid substitutions on protein function. Annu Rev Genomics Hum Genet. 2006;7:61-80.

21. Adzhubei IA, Schmidt S, Peshkin L, et al. A method and server for predicting damaging missense mutations. Nat Methods. 2010;7(4): 248-249.

22. Kircher M, Witten DM, Jain P, O'Roak BJ, Cooper GM, Shendure J. A general framework for estimating the relative pathogenicity of human genetic variants. Nat Genet. 2014:46(3):310-315.

23. Lek M, Karczewski KJ, Minikel EV, et al; Exome Aggregation Consortium. Analysis of protein-coding genetic variation in 60,706 humans. Nature. 2016;536(7616):285-291.

24. Samocha KE, Robinson EB, Sanders SJ, et al. A framework for the interpretation of de novo mutation in human disease. Nat Genet. 2014;46(9): 944-950.

25. Baker KE, Parker R. Nonsense-mediated mRNA decay: terminating erroneous gene expression. Curr Opin Cell Biol. 2004;16(3):293-299.

26. Speca DJ, Ogata G, Mandikian D, et al. Deletion of the Kv2.1 delayed rectifier potassium channel leads to neuronal and behavioral hyperexcitability. Genes Brain Behav. 2014;13(4):394-408.

27. Weckhuysen S, Mandelstam S, Suls A, et al. KCNQ2 encephalopathy: emerging phenotype of a neonatal epileptic encephalopathy. Ann Neurol. 2012;71(1):15-25. 